\title{
A combination of parabolic and grid slope interpolation for 2D tissue displacement estimations
}

\author{
John Albinsson ${ }^{1}$ Åsa Rydén Ahlgren ${ }^{2}$ Tomas Jansson ${ }^{3,4} \cdot$ Magnus Cinthio $^{1}$
}

Received: 18 April 2016 / Accepted: 26 October 2016 / Published online: 11 November 2016

(C) The Author(s) 2016. This article is published with open access at Springerlink.com

\begin{abstract}
Parabolic sub-sample interpolation for 2D blockmatching motion estimation is computationally efficient. However, it is well known that the parabolic interpolation gives a biased motion estimate for displacements greater than $|y .2|$ samples $(y=0,1, \ldots)$. Grid slope sub-sample interpolation is less biased, but it shows large variability for displacements close to $y .0$. We therefore propose to combine these sub-sample methods into one method (GS15PI) using a threshold to determine when to use which method. The proposed method was evaluated on simulated, phantom, and in vivo ultrasound cine loops and was compared to three sub-sample interpolation methods. On average, GS15PI reduced the absolute sub-sample estimation errors in the simulated and phantom cine loops by 14,8 , and $24 \%$ compared to sub-sample interpolation of the image, parabolic sub-sample interpolation, and grid slope sub-sample interpolation, respectively. The limited in vivo evaluation of estimations of the longitudinal movement of the common carotid artery using parabolic and grid slope sub-sample interpolation and GS15PI resulted in coefficient of variation (CV) values of $6.9,7.5$, and $6.8 \%$, respectively. The proposed method is computationally efficient and has low bias and variance. The method is another step toward a fast
\end{abstract}

John Albinsson

John.Albinsson@bme.lth.se

1 Department of Biomedical Engineering, Faculty of Engineering, Lund University, Ole Römers väg 3, 22100 Lund, Sweden

2 Department of Medical Imaging and Physiology, Skåne University Hospital, Lund University, Malmö, Sweden

3 Clinical Sciences Lund, Biomedical Engineering, Lund University, Lund, Sweden

4 Medical Services, Skåne University Hospital, Lund, Sweden and reliable method for clinical investigations of longitudinal movement of the arterial wall.

Keywords Ultrasound · Sub-sample estimation · Block matching $\cdot$ Speckle tracking $\cdot$ In silico $\cdot$ In vivo

\section{Introduction}

Tissue motion measurements using ultrasound can provide functional information about the tissue of interest and have attracted attention for various applications such as the evaluation of cardiac [14, 21, 22, 27, 32], vascular [4, 5, 19, $28]$, and skeletal muscle $[9,18,30]$ function.

One vascular application of interest is the measurement of the longitudinal movement of the arterial wall, i.e., the motion along the arteries $[12,17,35,36]$. In large arteries, the displacement is greatest in the layers closest to the lumen - the intima-media complex-and is of the same magnitude as the diameter change [13] (Fig. 1). The outer layer-the adventitia — shows the same basic pattern of movement, but the displacement is smaller, thereby demonstrating the presence of previously unknown substantial shear strain and thus shear stress, intramurally [13, 23, 33, 46]. Recent studies have reported that the amplitude of the longitudinal displacement of the arterial wall is reduced in patients with carotid plaques, suspected coronary artery disease, type 2 diabetes [38-40,42, 45], and periodontal disease [47], suggesting that the longitudinal movement of the arterial wall might prove to be a valuable marker for future risk of cardiovascular disease. Furthermore, in a study on the porcine carotid artery, we recently reported that longitudinal movement and intramural shear strain undergo profound changes in response to the important endogenous hormones adrenalin and noradrenalin [2]. 


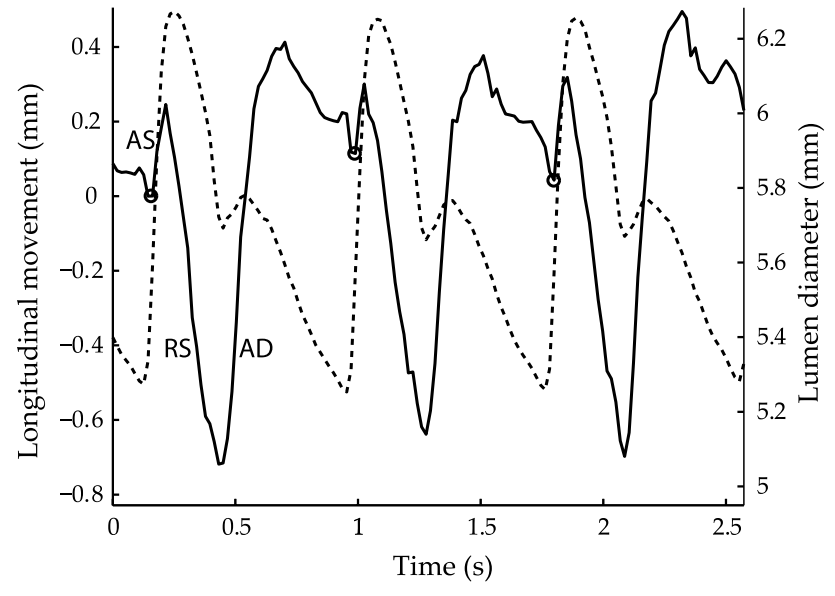

Fig. 1 Longitudinal movement (solid line) of the intima-media complex of the far wall and the corresponding diameter change (dashed line) in the common carotid artery of a 29 -year-old female during three cardiac cycles. For longitudinal movement, a positive deflection denotes movement in the direction of blood flow. The small circles mark the onset of an antegrade movement in early systole (AS). The distinct antegrade movement is followed by a distinct retrograde movement in systole (RS) and a second distinct antegrade movement in early diastole $(\mathrm{AD})$

These findings might have important implications for vascular disease both in the short- and long term and might constitute a link between mental stress and cardiovascular disease [2].

In order to calculate high-resolution motion estimates using block matching, three components are needed. (1) A search method is needed to determine which blocks the kernel should be compared to in order to find the most similar block [24]. (2) A method is needed to determine similarity [20] by calculating evaluation metric values between the kernel and the compared blocks. The value can be either the maximum likeness, e.g., the normalized crosscorrelation [37], or the minimum difference, e.g., the sum of the absolute difference [7]. (3) A sub-sample estimation method is needed to determine movements at sub-sample accuracy. The sub-sample estimation method can be one of the following three subgroups: (a) interpolation of the data points [31], (b) interpolation of evaluation metric values [10], or (c) analytically solving the min/max problem for the evaluation metric values [10]. The number of calculations needed for the three subgroups show that for the average method of each group the computational load is highest with (a) and lowest with (c). The motion estimations can be conducted with the ultrasound data in any of several forms, e.g., B-mode, radio-frequency, or after a Fourier transformation.

During the last decade, several tracking methods based on block matching have been developed to measure longitudinal movement of the arterial wall in ultrasound cine loops
$[3,12,13,15,34,41,44,45]$. The most common method to obtain sub-sample estimations is the use of image interpolation. Interpolation of the image gives good sub-sample estimations but is very time-consuming. Albinsson et al. [3] fitted three evaluation metric values with a parabolic function, which is a computationally efficient method to determine sub-sample displacements. However, it is well known that the parabolic function gives a biased estimation for displacements greater than $|y .2|$ samples $(y=0,1$, ...) [8]. Another sub-sample method, grid slope interpolation [16], gives fast unbiased motion estimates, but it has a large variability of the motion estimates for displacements close to $y .0$ samples (see also below). Considering that the drawbacks of the two sub-sample methods occur at different sub-sample displacements, a possible solution is to combine the two sub-sample methods. We therefore propose a new method, from now on denoted GS15PI, in which the sub-sample displacement is first estimated with a parabolic function. If the absolute sub-sample estimation is greater than a threshold (chosen to be 0.15 ), the sub-sample estimate is recalculated by grid slope interpolation.

The aim of this work was to evaluate the new sub-sample estimation method and to compare its performance to three sub-sample estimation methods: sub-sample interpolation of the image, parabolic sub-sample interpolation, and grid slope sub-sample interpolation. The evaluations were conducted on simulated and phantom ultrasound cine loops consisting of both B-mode data and radio-frequency data using different settings for the signal-to-noise ratio, velocity, and kernel size. Also, data from an in vivo study of the longitudinal displacement of the common carotid artery in healthy humans were used to evaluate the methods.

\section{Materials and methods}

Ultrasound is a modality based on reflected acoustical waves. The detected oscillating signals are beamformed and saved as radio-frequency (RF) data. A brightness mode (B-mode) image is created from the RF data by envelope detection and scan conversion. In the conversion into B-mode data, the RF data are normally down-sampled in the axial direction and displayed on a logarithmic scale. Thus, the two data types will typically have the same lateral sample distance, whereas the RF data will have shorter axial sample distance. The data points in RF signals are typically called "samples" because they are sampled from the acoustical waves, and the B-mode data points are called "pixels" because they represent the intensity data in an image.

Throughout this text, the word "sample" should be read as "sample and/or pixel" because the effects described are the same for both RF and B-mode data. 


\subsection{Ultrasound cine loops}

Ultrasound cine loops of three types of objects were used: a simulated object, a phantom object, and the far wall of the common carotid artery in vivo.

Ultrasound simulations were created using Field II [25, 26] running under MATLAB R2013a (The MathWorks, Inc., Natick, MA, USA). The settings used in the simulations are presented in Table 1. The in silico model consisted of a body of scatterers with random distribution and scatter power that was displaced a set distance between two images. The cine loops were divided into three groups

Table 1 Settings in Field II for the in silico cine loops

\begin{tabular}{ll}
\hline Width of element & $0.215 \mathrm{~mm}$ \\
Height of element & $6 \mathrm{~mm}$ \\
Distance between elements & $0.030 \mathrm{~mm}$ \\
$\begin{array}{l}\text { Number of elements in transmit/ } \\
\text { receive }\end{array}$ & 64 \\
Focus on transmission (fixed focal & $40 \mathrm{~mm}$ \\
$\quad$ point) & \\
Focus on receiving & Dynamic focusing \\
Elevational focus (acoustic lens) & $18 \mathrm{~mm}$ \\
Center frequency & $6 \mathrm{MHz}$ \\
Simulated transducer & LA523 (Esaote SpA, Florence, \\
& Italy) \\
Speed of sound & $1540 \mathrm{~m} / \mathrm{s}$ \\
Sampling rate & $100 \mathrm{MHz}$ \\
Number of scan lines & 128 \\
Size of phantom & $40 \times 50 \times 10 \mathrm{~mm}^{3}$ \\
$\quad$ (width $\times$ height $\times$ depth) & \\
Number of scatterers & 20,000 \\
\hline
\end{tabular}

according to the direction of the displacement: horizontal, vertical, or diagonal $\left(45^{\circ}\right)$. The movement of the scatterers was $(0.1 ; 0.3 ; 0.5 ; 0.7 ; 0.9 ; 1.2 ; 1.6 ; 2.0 ; 2.4 ; 2.8)$ pixels per image in all three groups. From each simulation, three cine loops were created with different levels of signal-to-noise ratio (SNR) by adding white noise to the RF data: no noise, SNR $21 \mathrm{~dB}$, and SNR $16 \mathrm{~dB}$. The RF data were down-sampled by a factor of 16 in the vertical direction during the scan conversion into B-mode data. The settings allowed a pixel density in the B-mode images of $8.1 \mathrm{pixel} / \mathrm{mm}$ axially and $4.1 \mathrm{pixe} / \mathrm{mm}$ laterally. Motion estimations were conducted using both the RF data and the B-mode data.

Phantom data were collected using both a research ultrasound machine, an Ultrasound Advanced Open Platform (ULAOP) [43] (University of Florence, Italy) equipped with a 4- to 13-MHz linear transducer (LA523, Esaote SpA, Florence, Italy), and a commercial ultrasound machine, a Philips EPIQ 7 equipped with a 3- to 12-MHz linear transducer (Philips Medical Systems, Bothell, WA, USA). Both B-mode data and RF data (down-sampled by a factor of 8 during the scan conversion) were available from ULAOP. The pixel density in the B-mode images was 8.1 pixels/ $\mathrm{mm}$ axially and 4.1 pixels $/ \mathrm{mm}$ laterally (the same as the in silico data). Only B-mode data in the DICOM format were available from the Philips EPIQ 7. The pixel density was 21.5 pixels $/ \mathrm{mm}$ both axially and laterally. Settings were chosen to obtain a frame rate close to $50 \mathrm{~Hz}$ using the highest line density, and persistence was turned off in order to avoid averaging between images. The phantom (a sponge) was moved in a water bath at velocities in the range of $2-15 \mathrm{~mm} / \mathrm{s}$ in steps of $1 \mathrm{~mm} / \mathrm{s}$ both purely laterally and diagonally within the scan-plane. B-mode data from in silico and phantom measurements are shown in Fig. 2.
Fig. 2 B-mode images from the three ultrasound sources: $\mathbf{a}$ in silico, b ULAOP, and c Philips EPIQ 7. The images each depict an area of $20 \times 15 \mathrm{~mm}^{2}$
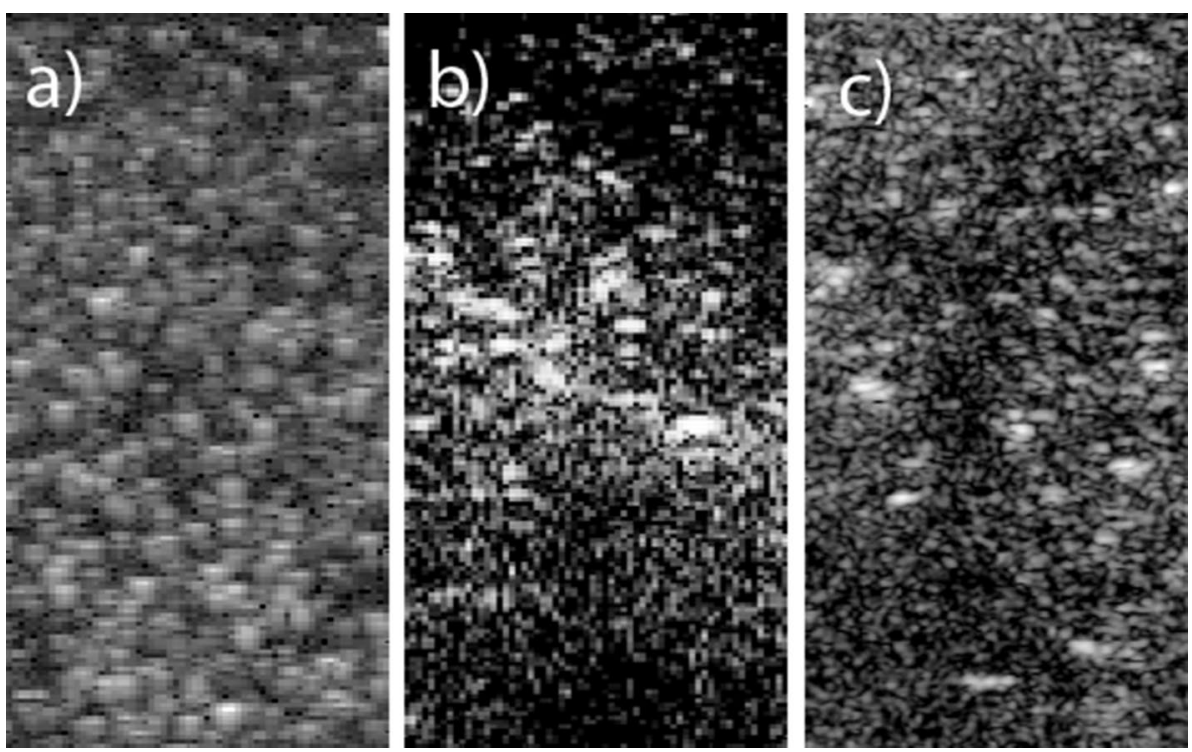
Table 2 Parameters investigated and their different settings

\begin{tabular}{ll}
\hline Parameter & Setting \\
\hline Image data type & B-mode, RF (not Philips EPIQ 7) \\
Size of kernel: (B-mode in silico and ULAOP) [pixels] & $7 \times 3,15 \times 7,23 \times 11$ \\
(B-mode Philips EPIQ 7) [pixels] & $17 \times 15,37 \times 15,57 \times 55$ \\
(RF in silico) [samples] & $112 \times 3,240 \times 7,368 \times 11$ \\
(RF ULAOP) [samples] & $56 \times 3,120 \times 7,184 \times 11$ \\
Size of kernel: all cine loops (mm) & $0.9 \times 0.7,1.8 \times 1.7,2.8 \times 2.7$ \\
Noise-SNR & $16 \mathrm{~dB}, 21 \mathrm{~dB}$, no noise \\
Velocity-direction & Vertical (not phantom), diagonal, horizontal \\
Velocity-in silico (pixels/frame) & $0.1,0.3,0.5,0.7,0.9,1.2,1.6,2.0,2.4,2.8$ \\
Velocity-phantom (mm/s) & $2,3,4,5,6,7,8,9,10,11,12,13,14,15$ \\
\hline
\end{tabular}

The right common carotid artery of 40 healthy volunteers (aged 20-69 years) was examined after at least 10 min of rest in a supine position using a Philips IU 22 equipped with a 5- to 12-MHz linear array transducer (Philips Medical Systems, Bothell, WA, USA). All volunteers gave informed consent according to the Helsinki Declaration, and the study was approved by the Ethics Committee of Lund University. Two cine loops were acquired for each volunteer. Settings were chosen to obtain a frame rate close to $50 \mathrm{~Hz}$ using the highest line density, and persistence was turned off in order to avoid averaging between images. DICOM data were exported for off-line motion estimations of the far wall of the common carotid artery. The pixel density was 21.5 pixels $/ \mathrm{mm}$ both axially and laterally.

\subsection{Methods for motion estimation}

A tracking scheme for $2 \mathrm{D}$ motion estimation was implemented using a full-search method and the sum of the absolute difference as the evaluation metric. The sub-sample positions were determined using four different methods: (1) interpolation of the image data values using cubical splines (CUBIC), (2) parabolic interpolation (PI), (3) modified grid slope interpolation (GSmod), and (4) the proposed method (GS15PI).

\subsubsection{Search method}

The full-search method searched for the best matching block among all possible blocks within a region of interest using the sum of the absolute difference [7] as the evaluation metric. In the B-mode, the size of the region of interest was the size of the kernel +10 samples both axially and laterally. In the RF data, the size of the region of interest was the size of the kernel +10 samples laterally, while it was (the size of the kernel +10$) \times 16$ samples axially. The kernel sizes used in silico and in the phantom measurements were $0.9 \times 0.7 \mathrm{~mm}^{2}, 1.8 \times 1.7 \mathrm{~mm}^{2}$, and $2.8 \times 2.7 \mathrm{~mm}^{2}$ (Table 2). The kernel sizes for the in vivo motion estimations were visually optimized for each volunteer. The chosen size was used in the two cine loops and for all sub-sample methods.

\subsubsection{Sub-sample estimation methods}

CUBIC was used to interpolate the ultrasound data 128 times both axially and laterally using cubical splines [29]. Only the data in the square of the current image centered on the position of the center of the block with the best similarity to the kernel were interpolated. The size of the square was two samples larger than the kernel both axially and laterally. The kernel was not interpolated but was compared to an equal number of interpolated samples obtained at every 128th sample of the interpolated segment. A full search was conducted in the entire interpolated square in order to find the best match at sub-sample resolution.

PI was used to estimate the sub-sample position by fitting a one-dimensional second-degree polynomial to three adjacent evaluation metric values [10] where the center value corresponded to the center position of the block with the best similarity to the kernel. The polynomial was fitted separately laterally and axially. The analytical solution of the polynomial gave the sub-sample estimation as:

$$
\Delta x=\frac{\alpha_{1}-\alpha_{3}}{2\left(\alpha_{1}+\alpha_{3}-2 \alpha_{2}\right)}
$$

where $\alpha_{2}$ (center), $\alpha_{1}$, and $\alpha_{3}$ (on each side of center) denote evaluation metric values and $\Delta x$ denotes the subsample part of the movement.

Grid slope interpolation [16] was used to estimate the sub-sample position by using four evaluation metric values that were calculated between the kernel and four blocks. Two blocks were from the current image-the block with the best similarity to the kernel and the one with the second best similarity. The other two blocks originated in the previous image at the position of the blocks used for the evaluation metric value in the current image. The sub-sample estimation was calculated by: 
$\Delta x=0.5\left(1-\frac{\alpha_{2}-\alpha_{i}}{\alpha_{2,0}-\alpha_{i, 0}}\right)$

where $\alpha_{2}$ (center) and $\alpha_{i}$ denote evaluation metric values in the current image, and $\alpha_{2,0}$ and $\alpha_{i, 0}$ denote the evaluation metric values in the previous image. This method was, in the original work, evaluated on B-mode data using the sum of the absolute difference on horizontal motion only [16].

To expand the utility of the grid slope interpolation methodology, we modified it by setting the variable $\alpha_{2,0}$ to zero and setting the variable $\alpha_{i, 0}$ to the evaluation metric value calculated between the best and second best matching blocks in the current image. This resulted in a method denoted GSmod. The sub-sample distance was estimated separately laterally and axially.

Our proposed method, GS15PI, was developed to take advantage of the best characteristics of PI and GSmod. GS15PI first estimates a sub-sample displacement using PI. If the estimated absolute sub-sample displacement is larger than 0.15 samples, the sub-sample estimation is recalculated using GSmod and accepted without further testing. The threshold of 0.15 samples was chosen after an empirical study on phantom movements (unpublished data).

\subsection{Evaluation of the motion estimations}

The settings that were investigated (summarized in Table 2) covered four sub-sample interpolation methods and five parameters-image data type, kernel size, noise (only for simulated data), direction of movement, and velocity of the object. In this work, two types of results were collected for 100 kernels for each combination of settings - the estimated displacements and the total estimation time used for the sub-sample estimations. Using in silico data, the different combinations noise levels, motion directions, and velocities resulted in 90 cine loops. Using three kernel sizes on each cine loop resulted in a total of 270 parameter settings to be evaluated for each combination of sub-sample method and image type. Using phantom data, there were 84 parameter settings.

A motion estimation error (per image) was defined as the geometrical difference between the set displacement and the estimated displacement, except in Table 4 where the lateral and axial components of the estimation errors were calculated separately. The mean value and standard deviation (SD) were estimated for each setting. The calculation time for a sub-sample motion estimation was measured separately from the search method. The time measurement was taken for 100 sub-sample motion estimations and averaged to give the mean time used for one sub-sample estimation.

The longitudinal movement of the common carotid artery in healthy humans at rest can show dramatically different multi-phasic patterns, even in subjects of similar age and gender [1, 13, 44]. An antegrade longitudinal movement in early systole is followed by a retrograde movement in systole (Fig. 1). The retrograde movement is the most distinct phase, present in all subjects, and is often the largest movement $[1,13,44]$. Therefore, we have chosen to use the magnitude of the retrograde movement in systole when comparing the PI, GSmod, and GS15PI subsample estimation methods in vivo. However, in some subjects, the antegrade movement in early systole is absent or very small, which makes the onset of the retrograde movement indistinct. In the present study, these subjects were excluded because the focus of this study was to evaluate the performance of the sub-sample estimation methods and not to evaluate the measurement of the phenomenon itself. Because the magnitude of the longitudinal displacement of the common carotid artery wall seems to decrease with distance from the heart [48], care was taken to perform the measurement at the same position in the two cine loops. The magnitude of the retrograde movement in systole was estimated over the course of 3-5 cardiac cycles per cine loop using a semiautomated method applied to the longitudinal movement curve (Fig. 1). The semiautomated method was initiated by a click on the position of the onset of the antegrade movement in systole. The evaluation of the subsample methods was performed by calculating the coefficient of variation (CV) [6] between the mean estimations of the magnitude of the retrograde movement in systole from two cine loops from the same volunteer.

\section{Results}

Using the combined in silico and phantom data, the mean estimation errors were smaller using GS15PI as compared to the other sub-sample methods. GS15PI on average reduced the estimation errors by $14 \%$ compared to CUBIC, by $8 \%$ compared to PI, and by $24 \%$ compared to GSmod. GS15PI also reduced the standard deviations by $12 \%$ compared to CUBIC, by $28 \%$ compared to PI, and by $2 \%$ compared to GSmod. However, there was a large variation in the results depending on the image source and data type in which the motion estimations were conducted (Table 3). In Table 3, the motion estimation errors were calculated using all kernel sizes, motion directions, speeds, and noise levels (where applicable).

The drawbacks of PI (bias in the motion estimations greater than $|y .2|$ ) and GSmod (variation in the motion estimations close to $y .0$ ) were clearly decreased for GS15PI. Figure 3 shows an example of the lateral component of the estimation errors using the following settings: in silico B-mode data, horizontal movement, SNR $21 \mathrm{~dB}$, and a kernel size of $1.8 \times 1.7 \mathrm{~mm}^{2}$. The bias of PI is visible as a 
Table 3 Mean estimation errors and corresponding standard deviation (SD) in $\mu \mathrm{m}$ for sub-sample estimation using in silico and phantom cine loops

\begin{tabular}{llllll}
\hline Image source & Data type & \multicolumn{5}{l}{ Sub-sample method } \\
\cline { 3 - 6 } & & CUBIC & PI & GSmod & GS15PI \\
\hline Philips EPIQ 7 & B-mode & $11(8.9)$ & $13(20)$ & $13(8.1)$ & $11(8.5)$ \\
ULAOP & B-mode & $85(200)$ & $95(200)$ & $90(200)$ & $92(200)$ \\
& RF & $78(190)$ & $77(200)$ & $67(200)$ & $72(200)$ \\
In silico & B-mode & $41(140)$ & $49(140)$ & $69(140)$ & $48(140)$ \\
& RF & $32(32)$ & $20(21)$ & $28(24)$ & $18(21)$ \\
\hline
\end{tabular}

The mean values were calculated over all settings for each data type and sub-sample method. The results are presented according to image data type (B-mode or RF data) and sub-sample estimation method (CUBIC-image interpolation, PI-parabolic interpolation, GSmod-modified grid slope interpolation, and GS15PI-our proposed method)

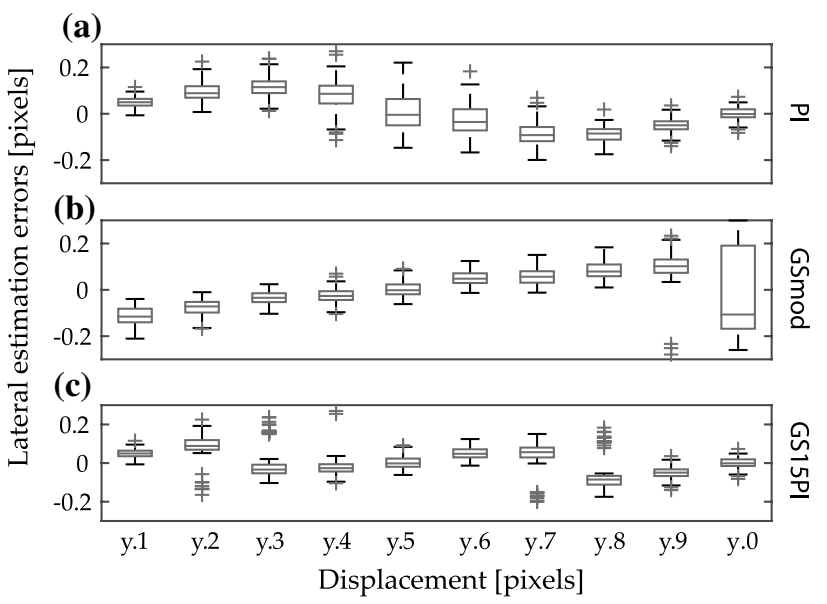

Fig. 3 Boxplot of the lateral motion estimation errors for a parabolic interpolation, b modified grid slope interpolation, and $\mathbf{c}$ our proposed method in relation to the set sub-sample movement. A total of 100 kernels were used for each velocity. The settings were in silico, B-mode data, horizontal movement, SNR $21 \mathrm{~dB}$, and a kernel size of $1.8 \times 1.7 \mathrm{~mm}^{2}$. From left to right, the velocities are 0.1, 1.2, 0.3 , $2.4,0.5,1.6,0.7,2.8,0.9$, and 2.0 pixels/image. The boxes indicate the lower and upper quartiles and the median. Outliers are indicated as crosses. Bias for each velocity can be seen as a deviation of the median from zero, and variation can be seen by the height of a box. Please note that 0.2 pixels $\approx 49 \mu \mathrm{m}$

deviation of the median error of each velocity from zero (Fig. 3a). The variation of GSmod is seen as the height of the box for $y .0$ (Fig. 3b). It can also be seen that GSmod had a small linear bias, which is consistent with the result presented by Geiman et al. [16] for the level of SNR in the image data. In Fig. 3c, the improvements in GS15PI can be seen as smaller variation at $y .0$ and median errors closer to zero. However, there are outliers at $y .2, y .3, y .7$, and $y .8$ (Fig. 3c).
The motion estimation errors were analyzed according to axial and lateral estimation errors (Table 4), motion direction (vertical, horizontal, and diagonal) (Table 5), kernel size (Table 6), and noise level (Table 7). As expected, axial errors were smaller than lateral errors, smaller errors were obtained using vertical motion direction than horizontal or diagonal motion directions, and better motion estimations were obtained by larger kernels and less noise. Also as expected, motion estimation using RF data gave smaller errors than using B-mode data, although there were exceptions for the small and medium-sized kernels using data from ULAOP (Table 6). The best results for all sub-sample methods over all image types were obtained using DICOM data from the commercial state-of-the-art Philips EPIQ 7 machine. One exception to the expected results was the larger motion estimation errors using CUBIC on RF data compared to the estimation errors using GS15PI (Tables 3, $4,5,6,7)$. There was no dependence between the motion estimation errors and the magnitude of the movement. Figure 4 shows an example of estimated accumulated displacements using the four sub-sample interpolation methods in a phantom at three different velocities and diagonal motion direction using the Philips EPIQ 7.

When using in silico data, the mean estimation time was longest for CUBIC followed by GSmod, GS15PI, and PI (Fig. 5). That CUBIC had a more than 27 times longer estimation time than the other methods was expected. When CUBIC was excluded, the sub-sample estimation time was about 1.1 times longer using RF data compared to using B-mode data.

The CV values and kernel sizes in vivo were calculated for 21 volunteers (aged 22-67 years) who had a distinct onset of retrograde movement in systole (Fig. 1). The used kernel sizes were in the range of $7 \times 13$ pixels to $11 \times 29$ pixels (which is equivalent to $0.29 \times 0.55 \mathrm{~mm}^{2}$ to $0.46 \times 1.22 \mathrm{~mm}^{2}$ ) with a mean kernel size of $7.1 \times 25$ pixels $\left(0.30 \times 1.05 \mathrm{~mm}^{2}\right)$. The $\mathrm{CV}$ values for the in vivo motion estimations of the retrograde movement in systole using PI, GSmod, and GS15PI were 6.9, 7.5, and 6.8\%, respectively. Figure 6 shows in vivo estimations of the longitudinal movement of the common carotid artery wall in one volunteer using the three sub-sample methods.

\section{Discussion}

We have presented a new ultrasound sub-sample motion estimation method, GS15PI, in which the best characteristics of two published methods, parabolic interpolation and grid slope interpolation, are combined to reduce their respective drawbacks of biased and noisy motion estimations (Fig. 3). The performance of GS15PI was evaluated on in silico, phantom, and in vivo cine loops. The new 
Table 4 Mean estimation errors and corresponding standard deviation (SD) values in $\mu \mathrm{m}$ separated according to their axial and lateral components using in silico and phantom cine loops

\begin{tabular}{lllcccc}
\hline Image source & Data type & Error component & \multicolumn{4}{l}{ Sub-sample method } \\
\cline { 3 - 6 } & & & CUBIC & PI & GSmod & GS15PI \\
\hline Philips EPIQ 7 & \multirow{2}{*}{ B-mode } & Axial & $-0.20(6.0)$ & $0.34(5.5)$ & $-1.6(8.1)$ & $0.26(6.0)$ \\
& & Lateral & $3.5(12)$ & $-1.1(23)$ & $2.0(13)$ & $2.1(12)$ \\
ULAOP & \multirow{2}{*}{ B-mode } & Axial & $4.3(99)$ & $4.8(100)$ & $3.7(100)$ & $4.8(100)$ \\
& & Lateral & $0.56(200)$ & $0.60(200)$ & $-0.79(200)$ & $0.88(200)$ \\
& \multirow{2}{*}{ RF } & Axial & $3.0(95)$ & $6.7(95)$ & $6.3(95)$ & $6.4(95)$ \\
& & Lateral & $-9.4(180)$ & $-8.7(190)$ & $-11(190)$ & $-8.7(190)$ \\
& \multirow{2}{*}{ Bn silico } & Axial & $15(62)$ & $19(62)$ & $24(62)$ & $17(62)$ \\
& \multirow{2}{*}{ R-mode } & Lateral & $35(130)$ & $41(130)$ & $60(130)$ & $41(130)$ \\
& Axial & $2.1(1.8)$ & $0.84(1.4)$ & $1.3(1.5)$ & $0.91(1.4)$ \\
& Lateral & $32(33)$ & $20(21)$ & $28(24)$ & $17(21)$ \\
\hline
\end{tabular}

The mean values were calculated over all settings. The results are presented according to image data type (B-mode or RF) and sub-sample estimation method (CUBIC - image interpolation, PI-parabolic interpolation, GSmod—modified grid slope interpolation, and GS15PI—our proposed method)

\begin{tabular}{|c|c|c|c|c|c|c|}
\hline \multirow[t]{2}{*}{ Image source } & \multirow[t]{2}{*}{ Data type } & \multirow[t]{2}{*}{ Motion direction } & \multicolumn{4}{|c|}{ Sub-sample method } \\
\hline & & & CUBIC & PI & GSmod & GS15PI \\
\hline \multirow[t]{2}{*}{ Philips EPIQ 7} & \multirow[t]{2}{*}{ B-mode } & $\mathrm{H}$ & $4.9(2.2)$ & $5.5(4.4)$ & $6.3(2.0)$ & $4.3(2.6)$ \\
\hline & & $\mathrm{D}$ & $5.6(3.0)$ & $6.4(4.2)$ & $6.8(3.1)$ & $6.2(3.2)$ \\
\hline \multirow[t]{4}{*}{ ULAOP } & \multirow[t]{2}{*}{ B-mode } & $\mathrm{H}$ & $13(8.3)$ & $19(7.3)$ & $18(9.3)$ & $16(8.0)$ \\
\hline & & $\mathrm{D}$ & $25(23)$ & $32(19)$ & $25(31)$ & $29(21)$ \\
\hline & \multirow[t]{2}{*}{$\mathrm{RF}$} & $\mathrm{H}$ & $18(12)$ & $14(5.0)$ & $9.3(5.7)$ & $11(4.6)$ \\
\hline & & $\mathrm{D}$ & $36(60)$ & $39(54)$ & $32(64)$ & $36(56)$ \\
\hline \multirow[t]{6}{*}{ In silico } & \multirow[t]{3}{*}{ B-mode } & $\mathrm{H}$ & $2.8(2.0)$ & $6.0(2.9)$ & $10(2.7)$ & $4.9(2.6)$ \\
\hline & & V & $4.5(3.4)$ & $6.4(2.8)$ & $23(3.5)$ & $6.5(4.7)$ \\
\hline & & $\mathrm{D}$ & $6.2(4.1)$ & $9.3(4.7)$ & $11(4.7)$ & $8.6(4.5)$ \\
\hline & \multirow[t]{3}{*}{$\mathrm{RF}$} & $\mathrm{H}$ & $12(9.0)$ & $11(5.7)$ & $6.8(5.6)$ & $9.3(6.1)$ \\
\hline & & V & $5.9(7.6)$ & $2.5(1.8)$ & $11(1.8)$ & $2.5(1.8)$ \\
\hline & & D & $11(8.4)$ & $5.1(2.7)$ & $5.4(2.0)$ & $3.5(2.1)$ \\
\hline
\end{tabular}

The results are presented according to image data type (B-mode or RF) and sub-sample estimation method (CUBIC - image interpolation, PI-parabolic interpolation, GSmod-modified grid slope interpolation, and GS15PI-our proposed method). The kernel size was $1.8 \times 1.7 \mathrm{~mm}^{2}$, and for the in silico data, the SNR was $21 \mathrm{~dB}$. Motion direction: $\mathrm{V}$ - vertical, $\mathrm{D}$ - diagonal, and $\mathrm{H}$-horizontal method performed well on both B-mode data and RF data, and the results were at the same level or better for both the magnitude of the motion estimation errors and the estimation time compared to image interpolation and parabolic and grid slope interpolation.

In general, our proposed method showed good stability in its motion estimations. Although the method did not always have the lowest motion estimation errors, the consistently low motion estimation errors resulted in an overall improvement compared to the motion estimation errors of the other sub-sample methods using in silico and phantom cine loops. A decrease in the standard deviation was also noted. This reliability in the motion estimations was also shown in the in vivo study. However, the outliers of the motion estimation errors at $y .2, y .3, y .7$, and $y .8$ shown in Fig. $3 \mathrm{c}$ indicate that the tuning of the method might not be optimal. GS15PI uses a threshold level of 0.15 samples to determine whether to use parabolic interpolation or grid slope interpolation, and a somewhat lower threshold level might have decreased the motion estimation error and variance at $y .2, y .3, y .7$, and $y .8$. Considering that the threshold was determined using phantom cine loops different from those used in this study (unpublished data), it is possible that the optimal threshold is dependent on some parameter in the cine loops. The implemented threshold was an on/off fixed-value version, and an adaptive version should also be considered in future work. 
Table 6 Mean estimation errors and corresponding standard deviation (SD) values in $\mu \mathrm{m}$ separated according to kernel size using in silico and phantom cine loops

\begin{tabular}{|c|c|c|c|c|c|c|}
\hline \multirow[t]{2}{*}{ Image source } & \multirow[t]{2}{*}{ Data type } & \multirow[t]{2}{*}{ Kernel size $\left(\mathrm{mm}^{2}\right)$} & \multicolumn{4}{|c|}{ Sub-sample method } \\
\hline & & & CUBIC & PI & GSmod & GS15PI \\
\hline \multirow[t]{3}{*}{ Philips EPIQ 7} & \multirow[t]{3}{*}{ B-mode } & $0.9 \times 0.8$ & $4.2(2.4)$ & $5.0(3.5)$ & $5.0(2.5)$ & $4.6(2.6)$ \\
\hline & & $1.8 \times 1.7$ & $3.7(2.0)$ & $4.3(2.8)$ & $4.6(2.0)$ & $4.1(2.2)$ \\
\hline & & $2.8 \times 2.7$ & $3.6(2.0)$ & $4.1(2.6)$ & $4.5(1.9)$ & $4.1(2.1)$ \\
\hline \multirow[t]{6}{*}{ ULAOP } & \multirow[t]{3}{*}{ B-mode } & $0.9 \times 0.8$ & $74(85)$ & $75(85)$ & $76(87)$ & $75(85)$ \\
\hline & & $1.8 \times 1.7$ & $16(15)$ & $21(13)$ & $17(21)$ & $20(14)$ \\
\hline & & $2.8 \times 2.7$ & $12(9.4)$ & $18(4.2)$ & $10(8.4)$ & $16(5.0)$ \\
\hline & \multirow[t]{3}{*}{$\mathrm{RF}$} & $0.9 \times 0.8$ & $83(100)$ & $81(100)$ & $82(110)$ & $81(110)$ \\
\hline & & $1.8 \times 1.7$ & $24(40)$ & $26(36)$ & $21(42)$ & $24(37)$ \\
\hline & & $2.8 \times 2.7$ & $12(8.6)$ & $16(2.6)$ & $10(5.7)$ & $13(4.0)$ \\
\hline \multirow[t]{6}{*}{ In silico } & \multirow[t]{3}{*}{ B-mode } & $0.9 \times 0.8$ & $43(67)$ & $45(65)$ & $46(68)$ & $44(67)$ \\
\hline & & $1.8 \times 1.7$ & $6.2(4.1)$ & $9.3(4.7)$ & $11(4.7)$ & $8.6(4.5)$ \\
\hline & & $2.8 \times 2.7$ & $4.1(2.6)$ & $7.4(3.1)$ & $9.9(2.3)$ & $6.7(2.2)$ \\
\hline & \multirow[t]{3}{*}{$\mathrm{RF}$} & $0.9 \times 0.8$ & $14(11)$ & $7.8(5.4)$ & $7.8(5.2)$ & $6.6(5.2)$ \\
\hline & & $1.8 \times 1.7$ & $11(8.4)$ & $5.1(2.7)$ & $5.4(2.0)$ & $3.5(2.1)$ \\
\hline & & $2.8 \times 2.7$ & $8.5(6.8)$ & $4.4(1.7)$ & $5.1(1.3)$ & $2.8(1.3)$ \\
\hline
\end{tabular}

The results are presented according to image data type (B-mode or RF) and sub-sample estimation method (CUBIC-image interpolation, PI-parabolic interpolation, GSmod-modified grid slope interpolation, and GS15PI-our proposed method). The motion direction was diagonal, and for the in silico data, the SNR was $21 \mathrm{~dB}$
Table 7 Mean errors and corresponding standard deviation (SD) values in $\mu \mathrm{m}$ separated according to noise level using in silico cine loops

\begin{tabular}{|c|c|c|c|c|c|}
\hline \multirow[t]{2}{*}{ Image type } & \multirow[t]{2}{*}{ Noise level } & \multicolumn{4}{|c|}{ Sub-sample method } \\
\hline & & CUBIC & PI & GSmod & GS15PI \\
\hline \multirow[t]{3}{*}{ B-mode } & SNR $16 \mathrm{~dB}$ & $9.3(6.7)$ & $12(6.1)$ & $16(6.8)$ & $11(6.6)$ \\
\hline & SNR $21 \mathrm{~dB}$ & $6.2(4.1)$ & $9.3(4.7)$ & $11(4.7)$ & $8.6(4.5)$ \\
\hline & No noise & $5.6(3.7)$ & $9.1(4.4)$ & $7.6(4.2)$ & $7.9(4.0)$ \\
\hline \multirow[t]{3}{*}{ RF } & SNR $16 \mathrm{~dB}$ & $12(9.0)$ & $4.8(2.8)$ & $9.6(2.7)$ & $4.8(2.6)$ \\
\hline & SNR $21 \mathrm{~dB}$ & $11(8.4)$ & $5.1(2.7)$ & $5.4(2.0)$ & $3.5(2.1)$ \\
\hline & No noise & $10(8.3)$ & $5.3(2.6)$ & $3.6(1.6)$ & $3.3(1.9)$ \\
\hline
\end{tabular}

The results are presented according to image data type (B-mode or $\mathrm{RF}$ ) and sub-sample estimation method (CUBIC-image interpolation, PI-parabolic interpolation, GSmod-modified grid slope interpolation, and GS15PI-our proposed method). The motion direction was diagonal, and the kernel size was $1.8 \times 1.7 \mathrm{~mm}^{2}$

The results were mostly as expected when analyzing the effects of the different parameters (Tables 4, 5, 6, 7). In line with other studies, the motion estimation errors decreased when the information in the kernel increased with kernel size. It is also known that less noise results in smaller motion estimation errors due to decreased rates of change in the speckle pattern. Different rates of change in the speckle pattern are also the likely cause for the differences in motion estimation errors between the three motion directions. A dependency between the actual distance between the sampled data and the size of the motion estimation errors was observed, i.e., a longer distance between samples resulted in larger motion estimation errors. No dependency between the velocity and the motion estimation errors was seen. This might be due to the limited change in the speckle pattern because we had relatively short movements per frame, and our intention was to have in-plane movements.

The motion estimation errors using CUBIC on RF data were larger than expected when compared to the other subsample methods (Tables 3, 4, 5, 6, 7). Because CUBIC performed well using B-mode data, the possibility of an implementation error was unlikely. In order to reduce the computation time during sub-sample estimation, we did not interpolate the kernel. This could be a possible explanation for the increase in these motion estimation errors, and further studies are needed to determine whether this is the case.

A limitation in this study was the absence of tests concerning strain and shearing in the simulations and in the phantom cine loops. Thus, how these motions affect the motion estimation errors of GS15PI compared to the other methods can only be speculated. However, the motion estimation errors using the in vivo cine loops, which incorporate a low level of strain in the investigated area, indicate that GS15PI has some robustness to strain. Further studies are needed to evaluate the influence of higher levels of strain and shearing when using GS15PI. Another limitation in our in vivo evaluation was the exclusion of CUBIC in the motion estimations. 

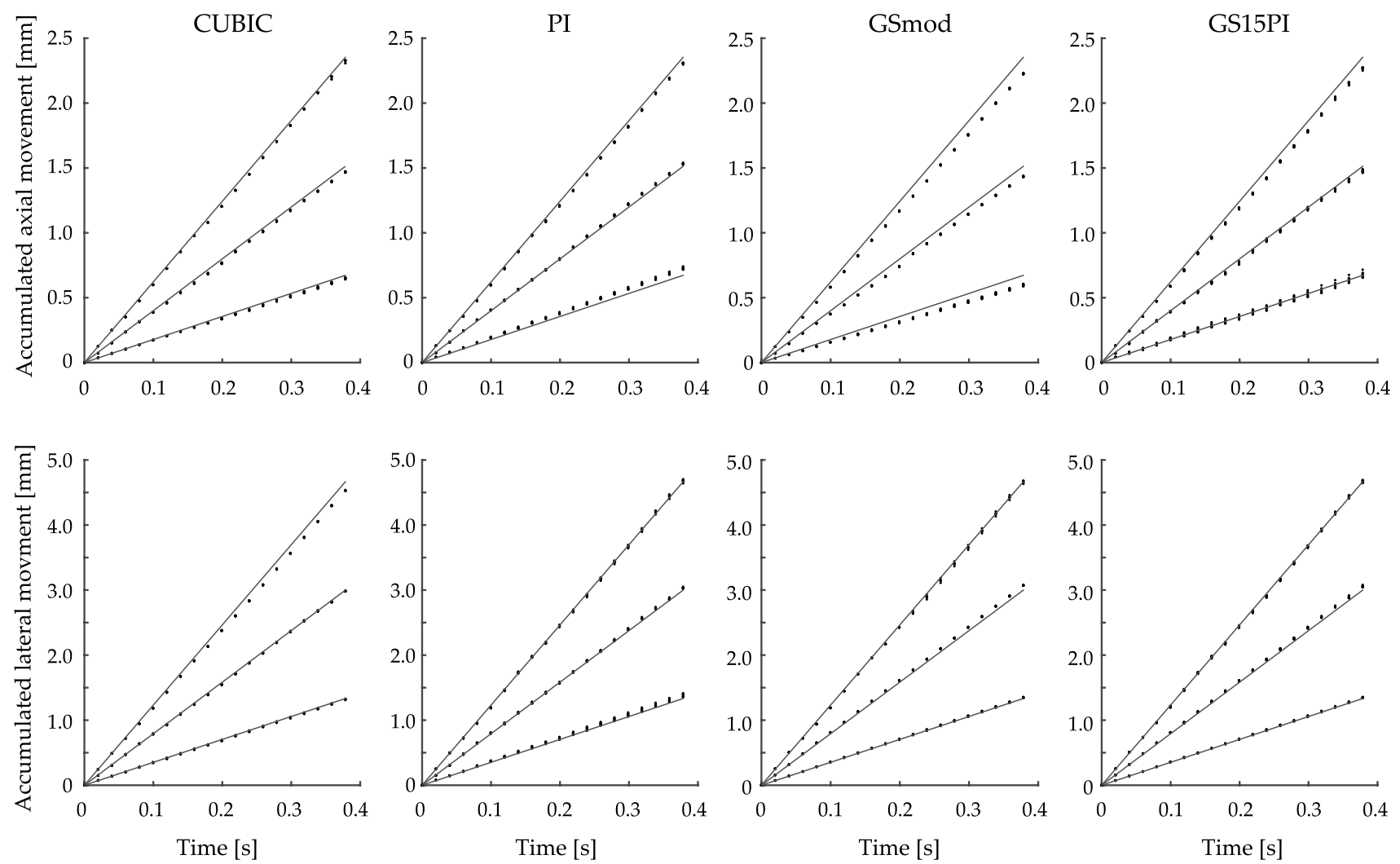

Fig. 4 Example of motion estimations in a phantom. The black lines indicate the set accumulated displacement for the diagonal motions at 3,8 , and $13 \mathrm{~mm} / \mathrm{s}$. The panels in the two rows show the accumulated

axial and lateral motion estimations of nine kernels using CUBICimage interpolation, PI-parabolic interpolation, GSmod-modified grid slope interpolation, and GS15PI—our proposed method

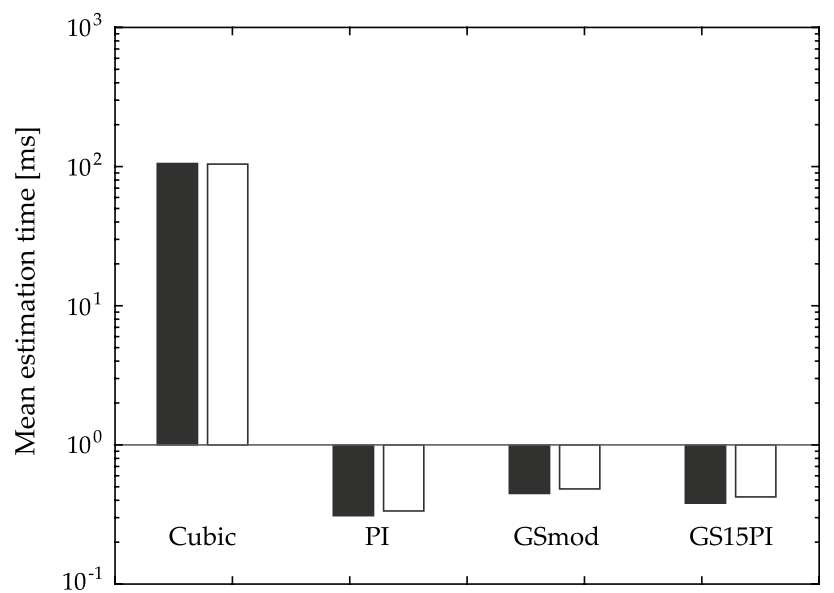

Fig. 5 Mean time to perform one estimation using the evaluated subsample methods on in silico cine loops for B-mode and RF data. The estimation times are presented according to image data type (B-mode and RF) and sub-sample estimation method (CUBIC-image interpolation, PI-parabolic interpolation, GSmod-modified grid slope interpolation, and GS15PI-our proposed method)

As expected, the computation time for CUBIC was much longer than the other sub-sample methods (approximately 27 times longer) because CUBIC involves many more calculations. This large difference has to be considered when the differences in the motion estimations are small. Please note that the difference in the computation time between the GS15PI and PI or GSmod methods was on the order of $1 / 10$ of a millisecond.

The purpose of the in vivo study in this work was to compare GS15PI to parabolic and grid slope interpolation of in vivo data. Therefore, we wanted a dataset in which the effect of the sub-sample method was dominant and thus we wanted to minimize other sources of error such as the time for the onset of the movement. Therefore, we restricted the comparison to volunteers with a distinct retrograde movement. The in vivo results in this study show promising results with $\mathrm{CV}$ values of $7 \%$; however, comparisons with other studies should be made with care. The selection of the volunteers in the present study improves our results 

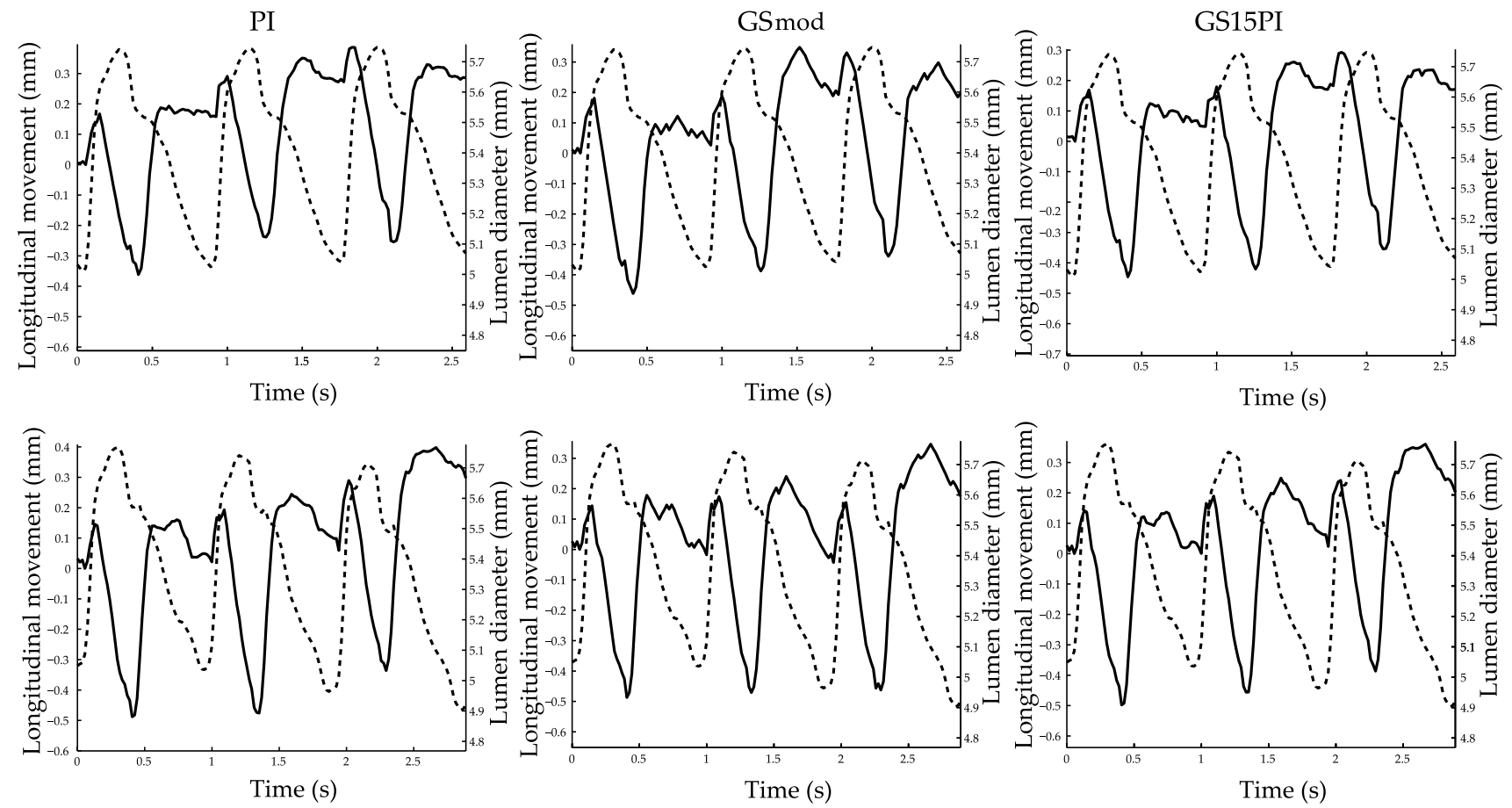

Fig. 6 Longitudinal movement (solid line) of the intima-media complex of the far wall and the corresponding diameter change (dashed line) in the common carotid artery of a 34-year-old female during three cardiac cycles. The panels in the two rows show the motion estimations in two different cine loops of the same volunteer using PI-parabolic interpolation, GSmod-modified grid slope interpolation, and GS15PI-our proposed method

computationally efficient compared to image interpolation and has low bias compared to parabolic interpolation and low variance at $y .0$ compared to grid slope interpolation. The method is another step toward fast and reliable clinical investigations of longitudinal movement of the arterial wall.

Acknowledgements This study was supported by grants from the Swedish Research Council (Grant No. 2012-3552), the Swedish Foundation for International Cooperation in Research and Higher Education (STINT) (Grant No. IG2011-2056), the Medical Faculty of Lund University, and the Skåne County Council's Research and Development Foundation.

Open Access This article is distributed under the terms of the Creative Commons Attribution 4.0 International License (http://creativecommons.org/licenses/by/4.0/), which permits unrestricted use, distribution, and reproduction in any medium, provided you give appropriate credit to the original author(s) and the source, provide a link to the Creative Commons license, and indicate if changes were made.

\section{References}

1. Ahlgren ÅR, Cinthio M, Persson HW, Lindström K (2012) Different patterns of longitudinal displacement of the common carotid artery wall in healthy humans are stable over a fourmonth period. Ultrasound Med Biol 38:916-925 
2. Ahlgren ÅR, Cinthio M, Steen S, Nilsson T, Sjöberg T, Persson HW, Lindström K (2012) Longitudinal displacement and intramural shear strain of the porcine carotid artery undergo profound changes in response to catecholamines. Am J Physiol Heart Circ Physiol 302:H1102-H1115. doi:10.1152/ajpheart.00470.2011

3. Albinsson J, Brorsson S, Ahlgren ÅR, Cinthio M (2014) Improved tracking performance of lagrangian block-matching methodologies using block expansion in the time domain-in silico, phantom and in vivo evaluations using ultrasound images. Ultrasound Med Biol 40:2508-2520

4. Arndt JO, Klauske J, Mersch F (1968) Diameter of intact carotid artery in man and its change with pulse pressure. Pflügers archiv Eur J Physiol 301:230-240

5. Benthin M, Dahl P, Ruzicka R, Lindström K (1991) Calculation of pulse wave velocity using cross correlation-effects of reflexes in the arterial tree. Ultrasound Med Biol 17:461-469

6. Bland JM, Altman DG (1986) Statistical methods for assessing agreement between two methods of clinical measurement. Lancet 327:307-310

7. Bohs LN, Trahey GE (1991) A novel method for angle independent ultrasonic imaging of blood flow and tissue motion. IEEE Trans Biomed Eng 38:280-286

8. Boucher RE, Hassab JC (1981) Analysis of discrete implementation of generalized cross correlator. IEEE Trans Acoust Speech ASSP 29:609-611

9. Brorsson S, Nilsdotter A, Hilliges M, Sollerman C, Aurell Y (2008) Ultrasound evaluation in combination with finger extension force measurements of the forearm musculus extensor digitorum communis in healthy subjects. BMC Med Imaging 8:6

10. Cespedes EI, Huang Y, Ophir J, Spratt S (1995) Methods for estimation of subsample time delays of digitized echo signals. Ultrason Imaging 17:142-171

11. Cinthio M, Ahlgren ÅR (2010) Intra-observer variability of longitudinal displacement and intramural shear strain measurements of the arterial wall using ultrasound noninvasively in vivo. Ultrasound Med Biol 36:697-704

12. Cinthio M, Ahlgren ÅR, Jansson T, Eriksson A, Persson HW, Lindström K (2005) Evaluation of an ultrasonic echo-tracking method for measurements of arterial wall movements in two dimensions. IEEE Trans Ultrason Ferroelectr Freq Control 52:1300-1311

13. Cinthio M, Ahlgren ÅR, Bergkvist J, Jansson T, Persson HW, Lindström K (2006) Longitudinal movements and resulting shear strain of the arterial wall. Am J Physiol Heart C 291:H394-H402

14. Dhooge J, Konofagou E, Jamal F (2002) Two-dimensional ultrasonic strain rate measurement of the human heart in vivo. IEEE Trans Ultrason Ferroelectr Freq Control 49:281-287

15. Gastounioti A, Golemati S, Stoitsis J, Nikita KS (2011) Comparison of Kalman-filter-based approaches for block matching in arterial wall motion analysis from B-mode ultrasound. Meas Sci Technol 22:114008. doi:10.1088/0957-0233/22/11/114008

16. Geiman BJ, Bohs LN, Anderson ME, Breit SM, Trahey GE (2000) A novel interpolation strategy for estimating subsample speckle motion. Phys Med Biol 45:1541-1552

17. Golemati S, Sassano A, Lever MJ, Bharath AA, Dhanjil S, Nicolaides AN (2003) Carotid artery wall motion estimated from B-mode ultrasound using region tracking and block matching. Ultrasound Med Biol 29:387-399

18. Grönlund C, Claesson K, Holtermann A (2013) Imaging twodimensional mechanical waves of skeletal muscle contraction. Ultrasound Med Biol 39:360-369

19. Hansen HHG, Lopata RGP, Idzenga T, de Korte CL (2010) An angular compounding technique using displacement projection for noninvasive ultrasound strain imaging of vessel crosssections. Ultrasound Med Biol 36:1947-1956. doi:10.1016/j. ultrasmedbio.2010.06.008
20. Hein IA, O'Brien WDJ (1993) Current time-domain methods for assessing tissue motion by analysis from reflected ultrasound echoes-a review. IEEE Trans Ultrason Ferroelectr Freq Control 40:84-102

21. Herbots L, Maes F, D'Hooge J, Claus P, Dymarkowski S, Mertens P, Mortelmans L, Bijnens B, Bogaert J, Rademakers FE (2004) Quantifying myocardial deformation throughout the cardiac cycle: a comparison of ultrasound strain rate, grey-scale M-mode and magnetic resonance imaging. Ultrasound Med Biol 30:591-598

22. Honjo Y, Hasegawa H, Kanai H (2010) Two-dimensional tracking of heart wall for detailed analysis of heart function at high temporal and spatial resolutions. Jpn J Appl Phys 7:11-19

23. Idzenga T, Holewijn S, Hansen HHG, de Korte CL (2012) Estimating cyclic shear strain in the common carotid artery using radiofrequency ultrasound. Ultrasound Med Biol 38:2229-2237

24. Jakubowski M, Pastuszak G (2013) Block-based motion estimation algorithms-a survey. Opto Electron Rev 21:86-102

25. Jensen JA (1996) Field: a program for simulating ultrasound systems. Med Biol Eng Comput 34:351-353

26. Jensen JA, Svendsen NB (1992) Calculation of pressure fields from arbitrarily shaped, apodized, and excited ultrasound transducers. IEEE Trans Ultrason Ferroelectr Freq Control 39:262-267

27. Kanai H, Hasegawa H, Chubachi N, Koiwa Y, Tanaka M (1997) Noninvasive evaluation of local myocardial thickening and its color-coded imaging. IEEE Trans Ultrason Ferroelectr Freq Control 44:752-768

28. Kanai H, Hasegawa H, Ichiki M, Tezuka F, Koiwa Y (2003) Elasticity imaging of atheroma with transcutaneous ultrasoundpreliminary study. Circulation 107:3018-3021

29. Keys RG (1981) Cubic convolution interpolation for digital image processing. IEEE Trans Acoust Speech Signal Process ASSP 29:1153-1160

30. Korstanje J-WH, Selles RW, Stam HJ, Hovius SER, Bosch JG (2010) Development and validation of ultrasound speckle tracking to quantify tendon displacement. J Biomech 43:1373-1379

31. Lehmann TM, Gönner C, Spitzer K (1999) Survey: interpolation methods in medical image processing. IEEE Trans Med Imaging 18:1049-1075

32. McDicken WN, Sutherland GR, Moran CM, Gordon LN (1992) Colour Doppler velocity imaging of the myocardium. Ultrasound Med Biol 18:651-654

33. Nilsson T, Ahlgren ÅR, Jansson T, Persson HW, Nilsson J, Lindström K, Cinthio M (2010) A method to measure shear strain with high-spatial-resolution in the arterial wall non-invasively in vivo by tracking zero crossings of B-Mode intensity gradients. Proc IEEE Ultrason Symp. doi:10.1109/ULTSYM.2010.5935442

34. Numata T, Hasegawa H, Kanai H (2007) Basic study on detection of outer boundary of arterial wall using its longitudinal motion. Jpn J Appl Phys 46:4900-4907

35. Persson M, Ahlgren ÅR, Eriksson A, Jansson T, Persson HW, Lindström K (2002) Non-invasive measurement of arterial longitudinal movement. Proc IEEE Ultrason Symp 2:1783-1786

36. Persson M, Ahlgren ÅR, Jansson T, Eriksson A, Persson HW, Lindström K (2003) A new non-invasive ultrasonic method for simultaneous measurements of longitudinal and radial arterial wall movements: first in vivo trial. Clin Physiol Funct I 23:247-251

37. Rosenfield A (1969) Picture processing by computer. Comput Surv 1:147-174

38. Soleimani E, Mokhtari-Dizaji M, Saberi H (2015) A novel noninvasive ultrasonic method to assess total axial stress of the common carotid artery wall in healthy and atherosclerotic men. J Biomech 48:1860-1867. doi:10.1016/j.jbiomech.2015.04.032

39. Svedlund S, Gan L-M (2011) Longitudinal common carotid artery wall motion is associated with plaque burden in man 
and mouse. Atherosclerosis 217:120-124. doi:10.1016/j. atherosclerosis.2011.02.046

40. Svedlund S, Eklund C, Robertsson P, Lomsky M, Gan L-M (2011) Carotid artery longitudinal displacement predicts 1-year cardiovascular outcome in patients with suspected coronary artery disease. Arterioscerl Thromb Vasc 31:1668-1674

41. Tat J, Au JS, Keir PJ, Macdonald MJ (2015) Reduced common carotid artery longitudinal wall motion and intramural shear strain in individuals with elevated cardiovascular disease risk using speckle tracking. Clin Physiol Funct I. doi:10.1111/ cpf. 12270

42. Tat J, Psaromiligkos IN, Daskalopoulou SS (2016) Carotid atherosclerotic plaque alters the direction of longitudinal motion in the artery wall. Ultrasound Med Biol 42:2114-2122. doi:10.1016/j.ultrasmedbio.2016.04.014

43. Tortoli P, Bassi L, Boni E, Dallai A, Guidi F, Ricci S (2009) An advanced open platform for ultrasound research. IEEE Trans Ultrason Ferroelectr Freq Control 56:2207-2216

44. Yli-Ollila H, Laitinen T, Weckström M, Laitinen TM (2013) Axial and radial waveforms in Common Carotid Artery: an advanced method for studying arterial elastic properties in ultrasound imaging. Ultrasound Med Biol 39:1168-1177

45. Zahnd G, Boussel L, Marion A, Durand M, Moulin P, Serusclat A, Vray D (2011) Measurement of two-dimensional movement parameters of the carotid artery wall for early detection of arteriosclerosis: a preliminary clinical study. Ultrasound Med Biol 37:1421-1429

46. Zahnd G, Boussel L, Serusclat A, Vray D (2011) Intramural shear strain can highlight the presence of atherosclerosis: a clinical in vivo study. Proc IEEE Ultrason Symp 1770-1773

47. Zahnd G, Maple-Brown LJ, O'Dea K, Moulin P, Celermajer DS, Skilton MR, Vray D, Sérusclat A, Alibay D, Bartold M, Brown A, Durand M, Jamieson LM, Kapellas K (2012) Longitudinal displacement of the carotid wall and cardiovascular risk factors: associations with aging, adiposity, blood pressure and periodontal disease independent of cross-sectional distensibility and intima-media thickness. Ultrasound Med Biol 38:1705-1715. doi:10.1016/j.ultrasmedbio.2012.05.004

48. Zahnd G, Balocco S, Sérusclat A, Moulin P, Orkisz M, Vray D (2015) Progressive attenuation of the longitudinal kinetics in the common carotid artery: preliminary in vivo assessment. Ultrasound Med Biol 41:339-345. doi:10.1016/j. ultrasmedbio.2014.07.019

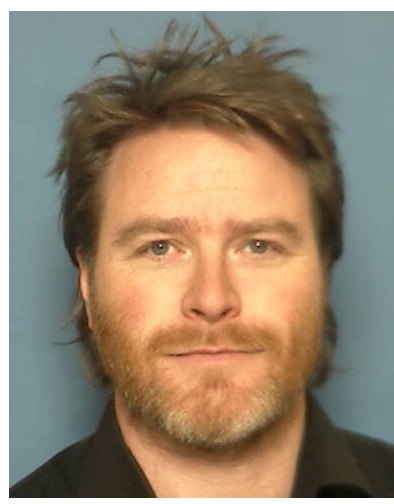

John Albinsson is a Ph.D. student at the Department of Biomedical Engineering, Lund University. His thesis is about tissue motion estimations in ultrasound images.

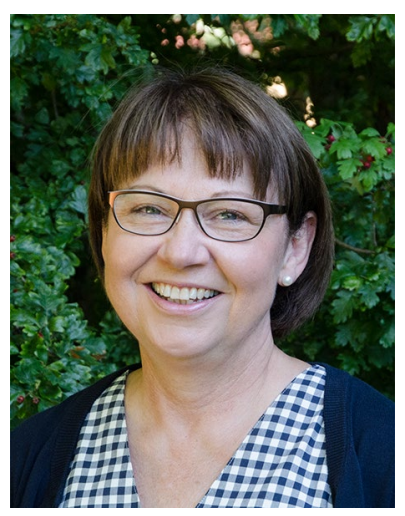

Åsa Rydén Ahlgren M.D., received her Ph.D. degree in 1998 from Lund University, Sweden. Her current research includes vascular mechanics and the longitudinal displacement of the arterial wall.

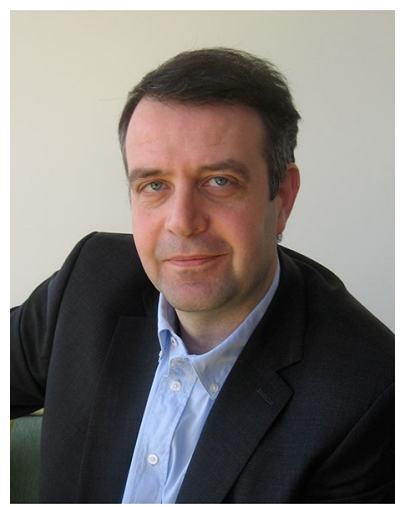

Tomas Jansson received his $\mathrm{Ph} . \mathrm{D}$. degree in 1999 from Lund University, Sweden. His current research includes magnetomotive ultrasound imaging and auditory perception of Doppler signals.

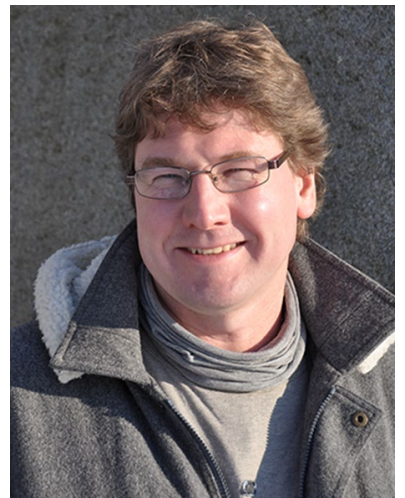

Magnus Cinthio received his Ph.D. degree in 2004 from Lund University, Sweden. His research interests include characterization of the arterial wall using ultrasound and magnetomotive ultrasound imaging. 